\title{
Space-qualified Piezo Based Deformable Mirror for future Instruments with Active Optics
}

Maximilian Freudling, Andreas Grzesik, Markus Erhard, Maximilian Gerhards, Sinje Leitz, et al.

Maximilian Freudling, Andreas Grzesik, Markus Erhard, Maximilian Gerhards, Sinje Leitz, Sven Verpoort, Ulrich Wittrock, Pascal Hallibert, "Space-qualified Piezo Based Deformable Mirror for future Instruments with Active Optics," Proc. SPIE 11852, International Conference on Space Optics - ICSO 2020, 1185231 (11 June 2021); doi: 10.1117/12.2599467

SPIE Event: International Conference on Space Optics - ICSO 2021, 2021, Online Only 


\section{International Conference on Space Optics-ICSO 2020}

Virtual Conference

30 March-2 April 2021

Edited by Bruno Cugny, Zoran Sodnik, and Nikos Karafolas
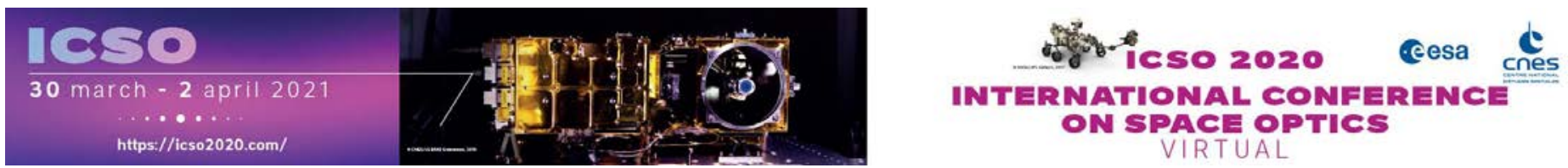

\section{Space-qualified Piezo Based Deformable Mirror for future Instruments with Active Optics}

\section{- esa issopocesalings denes}




\title{
Space-qualified Piezo Based Deformable Mirror for future Instruments with Active Optics
}

\author{
Maximilian Freudling ${ }^{\mathrm{a}}$, Andreas Grzesik ${ }^{\mathrm{a}}$, Markus Erhard ${ }^{\mathrm{a}}$, Maximilian Gerhards ${ }^{\mathrm{b}}$, Sinje Leitz ${ }^{\mathrm{b}}$, \\ Sven Verpoort ${ }^{\mathrm{b}}$, Ulrich Wittrock ${ }^{\mathrm{b}}$, Pascal Hallibert ${ }^{\mathrm{c}}$ \\ ${ }^{a}$ OHB System AG - Manfred-Fuchs-Str. 1, D-82234 Weßling, Germany \\ ${ }^{\mathrm{b}}$ Münster University of Applied Sciences - Stegerwaldstraße 39, 48565 Steinfurt, Germany \\ ${ }^{\mathrm{c}}$ European Space Agency - European Space Research and Technology Center, Noordwijk, The \\ Netherlands
}

\begin{abstract}
This paper presents the results of the technology development project "Enabling Technologies for Piezo-Based Deformable Mirrors in Active Optics Correction Chains" conducted by OHB System AG together with its partner Münster University of Applied Sciences (MUAS). The project was funded by ESA within their General Support Technology Programme (GSTP).

We address in this paper mainly the definition, flow-down and verification of the requirements for the Deformable Mirror (DM). The requirements were derived from a set of real space mission applications. The deformation of the mirror is performed by piezo-ceramic actuators in an unimorph configuration. The finally developed DM is able produce Zernike modes with a stroke of several tens of $\mu \mathrm{m}$ over a clear optical aperture of $50 \mathrm{~mm}$ in diameter. It underwent successfully a full environmental qualification campaign including thermal cycling, shock- and vibration testing, as well as exposure to proton and $\gamma$-ray radiation. Thermal and performance tests were performed in the temperature range from $100 \mathrm{~K}$ to $300 \mathrm{~K}$. Furthermore, the DM sustained all vibration (random $17.8 \mathrm{~g}$ RMS and sinus) and shock (300 g) testing. Thereby all criticalities which were identified a previous study have been overcome successfully.

A Technology Readiness Level (TRL) of 5 is reached, as the component has been validated in relevant environment. Based on the high level of maturity, this deformable mirror is now ready for the incorporation in future flight instruments. The achieved TRL of 5 is sufficient for the status of a PDR at payload level and gives thus a very good basis for all kinds of potential B2, C/D payload developments.
\end{abstract}

Keywords: Deformable Mirror, Active Optics, Space Qualification, Space Telescopes, Adaptive Optics

\section{INTRODUCTION}

For the next generation of optical space instruments with demands on improved performance and larger apertures, actively deformable mirrors for on-board wave-front and line of sight correction will play an important role. For this reason, ESA has supported the development of such a deformable mirror by means of a GSTP program. A piezo-based deformable mirror has to meet very challenging requirements, forcing to enable very high strokes and the correction of up to 12 Zernike coefficients on one side, but also to provide a very robust opto-mechanical and thermo-elastic design which is able to sustain high environmental loads on the other side.

In this project, OHB System AG collaborated with the Münster University of Applied Science (MUAS), who developed an earlier prototype of the DM in a previous study. Together a successful re-design of the DM was performed, reaching a full compliance to a revisited and updated set of requirements. The environmental and performance requirements were derived from real and typical earth observation and science missions. An important aspect described in this paper is how the optical requirements were derived, enabling the usage of the deformable mirror as a recurrent type of product for a large variety of space applications. Herby, the results of an ESA study on large monollithic mirros, the EUCLID mission and the Meteosat Third Generation Instrument for GEO, as well as LEO Hyperspectral Imager Mission EnMAP served as a basis. MTG and EnMAP Payloads have been developed by OHB. For EUCLID, OHB has designed and delivered the $\mathrm{CaLa}$ and the CoLa Lensassemblies for the NI-OA instrument [7][8][9]. The remaining part of this paper will present the full scope of the reached qualification. It will show, that a Technology Readiness Levels equal to TRL5 can be considered 
as the component has been validated in relevant environment. The very high level of maturity achieved for the developed deformable mirror provides a sound basis for an incorporation into future instrument baseline concepts (PDR level).

The newly developed mirror design is shown in Figure 1. A more detailed description of the DM design and performance characteristics is presented in another conference contribution by MUAS [2].
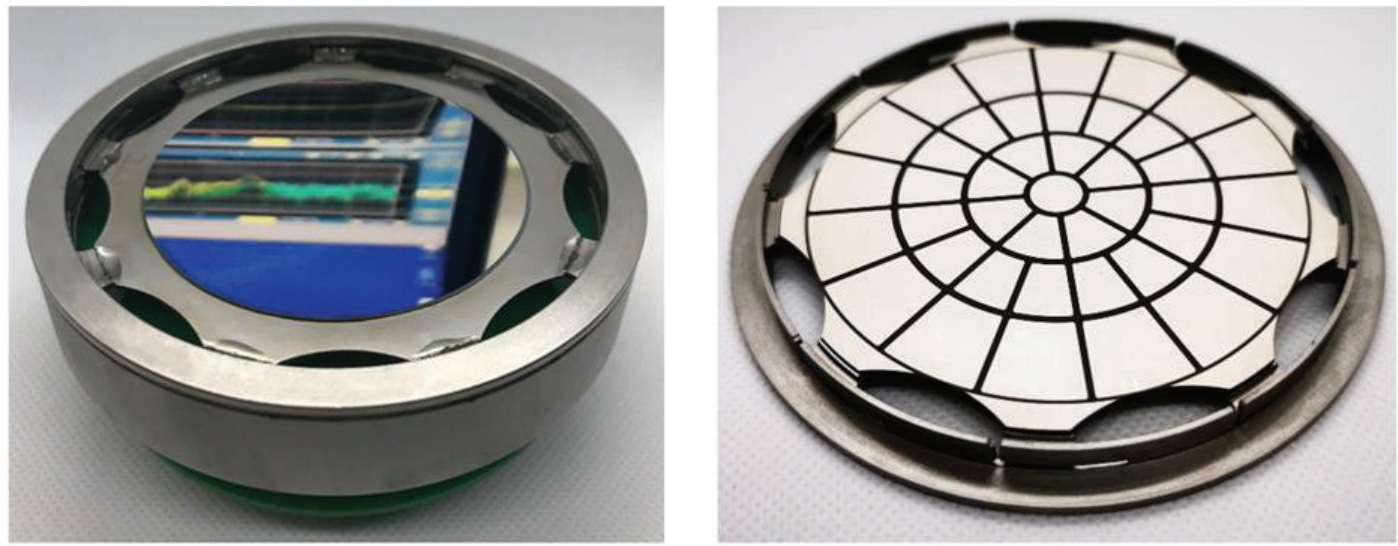

Figure 1. Deformable Mirror design with an isostatic mounting concept, front side (left) and rear side (right).

\section{REQUIREMENT CONSOLIDATION}

For the consolidation of the requirements, the specification from a previous GSTP study accomplished in 2015 was used as a starting point. This set of requirements was revisited and consolidated. For the consolidation of the requirements, OHB brought in experience from existing space instruments such as EUCLID, Meteosat Third Generation (MTG) and Environmental Mapping (EnMap) [7][8]. To derive the optical requirements, additional inputs from another ESA technology development studying aberrations of large light-weighted primary mirrors were used. This study was called "Active Correction Chain for Large Monolithic Mirror".

Special attention for the consolidation was put on the thermal and structural demands as well as the optical stroke. The consolidation of the structural dynamic requirements, namely random vibration and shock loads, were considered the most critical aspect for this project. The mechanical requirements were derived from ECSS standards and the experience of realistic load levels from various existing missions. The established thermal requirements cover a very wide range from typical temperatures in earth observation missions (LEO and GEO orbits), but go also down to cryogenic temperatures which might occur in scientific missions. There were no major changes in the specified temperatures compared to the previous project, thereby the thermal qualification from the previous project is still valid.

\subsection{Optical Requirements}

For the requirement derivation, the intention was to use available data from the alignment tolerance from various optical space instrument developments in order to get a better understanding of the typical stroke amplitudes needed for a comparable mission. The same order of magnitude is expected for in-orbit thermo-elastic deformations in these kind of instruments.

There are various applications for deploying a deformable mirror in a space optical instrument. For example when the deformable mirror is used to compensate deformations of a large primary mirror of a telescope due to gravity release or thermos-elastic effects. In this case, the deformable mirror should be placed at a conjugate plane of the primary mirror and the size ratio between primary and deformable mirror will directly limit the effectiveness of the optical correction. The deformable mirror concept under study in this project however aims at being a versatile tool, not linked to a too specific system design. A diameter of $50 \mathrm{~mm}$ is comparable to the accessible pupil planes of the typical programmes described above, was thus chosen for this activity.

Active tip tilt correction can be performed by an actuated mirror mount providing the advantage of not driving the actuators stroke requirements at the detriment of other conflicting requirements (correction of high-order Zernike polynomials etc.). Depending on the application, it can therefore be beneficial to put the main tip tilt function to an additional mechanism. 
For this project the required tilting was derived from the ESA large telescope study, which specifies a correction of $0.5 \mathrm{mrad}$. However, OHB assessment is that a $0.3 \mathrm{mrad}$ correction is also be sufficient. As mentioned before, assuming that large tip/tilt corrections are realized on a separate mechanism, so that this DM is rather used for fine tuning.

The following discussion applies to all Zernike terms. In order to assess what optical disturbances in a mid-class earth observation instrument can be expected and whether they can be corrected with a DM, analysis results from the mentioned programmes were taken into account. For the evaluation of the optical disturbances, it was assumed that only a rough placement of the optical components based on mechanical I/F characterizations would be done and the residual optical aberrations are then compensated by the DM. This is considered as an absolute worst case requirement, since analysis shows also that in-orbit deformations of the optical elements with the optical chain to be compensated by a DM would be much smaller. For information all four mirrors of the IRS front telescope were placed with an average accuracy of $100 \mu \mathrm{m}$. For this investigation, M4 of the telescope was considered to be a deformable mirror. The performance is evaluated using a Monte Carlo simulation with 1000 samples and the error budgets derived for MTG. The performance obtained are comparable and validate the potential approach. We observe that the strokes specified $(15 \mu \mathrm{m}$ for defocus and $5 \mu \mathrm{m}$ for the other Zernike) are very large compared to what would be required for such an application. From a comparison of the WFE values in $\mathrm{nm}$ rms and the calculated deformation amplitudes in the nm regime, a defocus requirement of $5 \mu \mathrm{m}$ amplitude is well suitable for a deformable mirror of that size.

In order to derive stroke requirements to compensate In-Orbit deformations of a large primary telescope mirror we used the following Korsch type telescope layout.

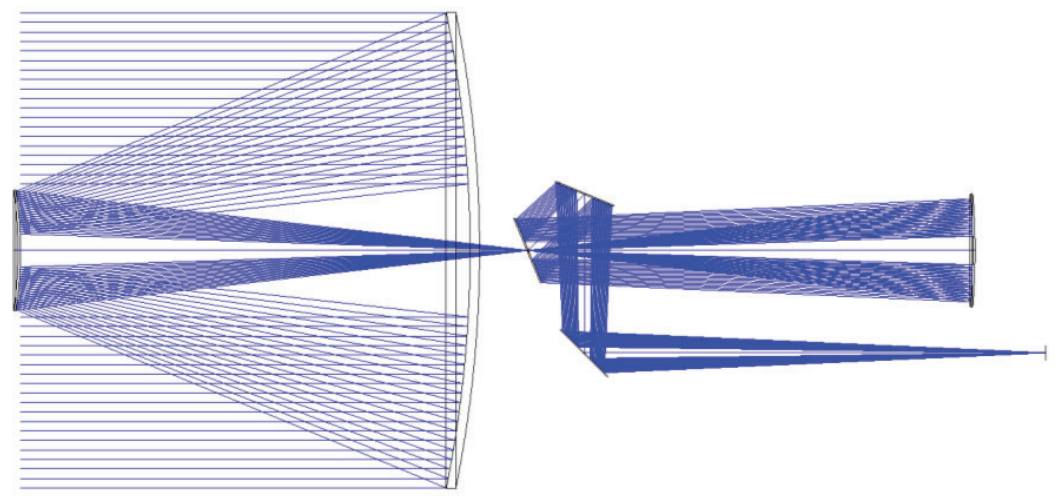

Figure 2. Korsch type telescope design for conceptual study on stroke requirements.

This telescope has the advantage of providing a location in the beam path where a deformable mirror will be conjugated to the primary mirror without the need for relay optics. However, it does not give much flexibility in the size of this conjugate plane. In the system under consideration, the diameter was $400 \mathrm{~mm}$.

Given this conjugation strategy, the strokes required to correct the modes are identical to the modes themselves. For a primary mirror of $3 \mathrm{~m}$ in diameter, and the deformable mirror size would need to be $400 \mathrm{~mm}$, given a $50 \mathrm{~mm} \mathrm{DM}$ (as considered in this study) the primary will be about $750 \mathrm{~mm}$ in diameter. In order to derive a requirement from the analyses for the further development a margin of $100 \%$ is considered. This shall ensure appropriate flexibility for further potential applications. The only exception is the astigmatism Z4 and Z5. Here the driving case is the MTG case for a not finally aligned (only placement) telescope which is considered as an absolute worst case (factor of six to the large telescope study). Therefore, there is not additional margin considered for Z4 and Z5. The consolidated requirement for the different Zernike Z4 to Z10 can be found in the table below.

Table 1. Required strokes for active correction.

\begin{tabular}{lr} 
Zernike Mode & Stroke \\
\hline Defocus & $5 \mu \mathrm{m}$ \\
Astig 3rd order $0^{\circ}$ & $3.2 \mu \mathrm{m}$ \\
Astig 3rd order $45^{\circ}$ & $3.2 \mu \mathrm{m}$ \\
Coma X 3rd order & $1.7 \mu \mathrm{m}$ \\
Coma Y 3rd order & $1.7 \mu \mathrm{m}$ \\
Spherical 3rd order & $0.35 \mu \mathrm{m}$ \\
Trifoil $0^{\circ}$ & $0.3 \mu \mathrm{m}$ \\
Trifoil 30 & $0.3 \mu \mathrm{m}$
\end{tabular}


The residual wave-front deviation needs to be defined as a function of the required system performance. The example of MTG-IRS can be used as described before. The value of 30nm PV for the DM in order to achieve an instrument WFE performance of ca. $80 \mathrm{~nm}$ RMS is reasonable. The un-powered mirror deformation of the DM will contribute to a reduction in the achievable stroke. An unpowered mirror deformation of $2 \mu \mathrm{m}$ still allows for $13 \mu \mathrm{m}$ strokes which is largely sufficient for the target applications.

\subsection{Structural Requirements}

As a starting point for the structural design of the DM, the quasi-static design loads were defined according to ECSS-EHB-32-26A. These are used at the beginning of the design phase as an envelope of the loads. To derive preliminary design loads for the DM, the following formula as shown below shall be used [5].

$$
\begin{gathered}
Q S_{D L}=\frac{70}{m^{0.3}} \\
Q S_{D L}: \text { preliminary } Q S \text { design load }[\mathrm{g}] \\
\text { m: assembly mass }[\mathrm{kg}]
\end{gathered}
$$

These loads were used to dimension structural parts, calculation preliminary interface forces, momentums and the primary notching. Using the formula with the mass of the current DM design $(\mathrm{m}=1.57[\mathrm{~kg}])$ results in a preliminary quasi-static load of $61.14[\mathrm{~g}]$ in all axes.

The sine loads were not considered critical for the DM with a mass of $1.57 \mathrm{~kg}$, since the first Eigen-frequency was higher than the maximum frequency of sine loads $(150 \mathrm{~Hz})$. For the same reason, the sine test was considered as being a quasistatic load test.

To derive and consolidate the requirement for the random vibrations the ECSS - Space Engineering, Testing was used [4]10. The basis for the load calculations is the mass of the DM of $1.57 \mathrm{Kg}$. The resulting loads are derived by using the mass and the formulas for units not located on external panels given in the cited ECSS. These calculated loads are shown in the figure below. In comparison, the similar requirements on the DM from the previous study are plotted for in-plane and out-of-plane random excitation.

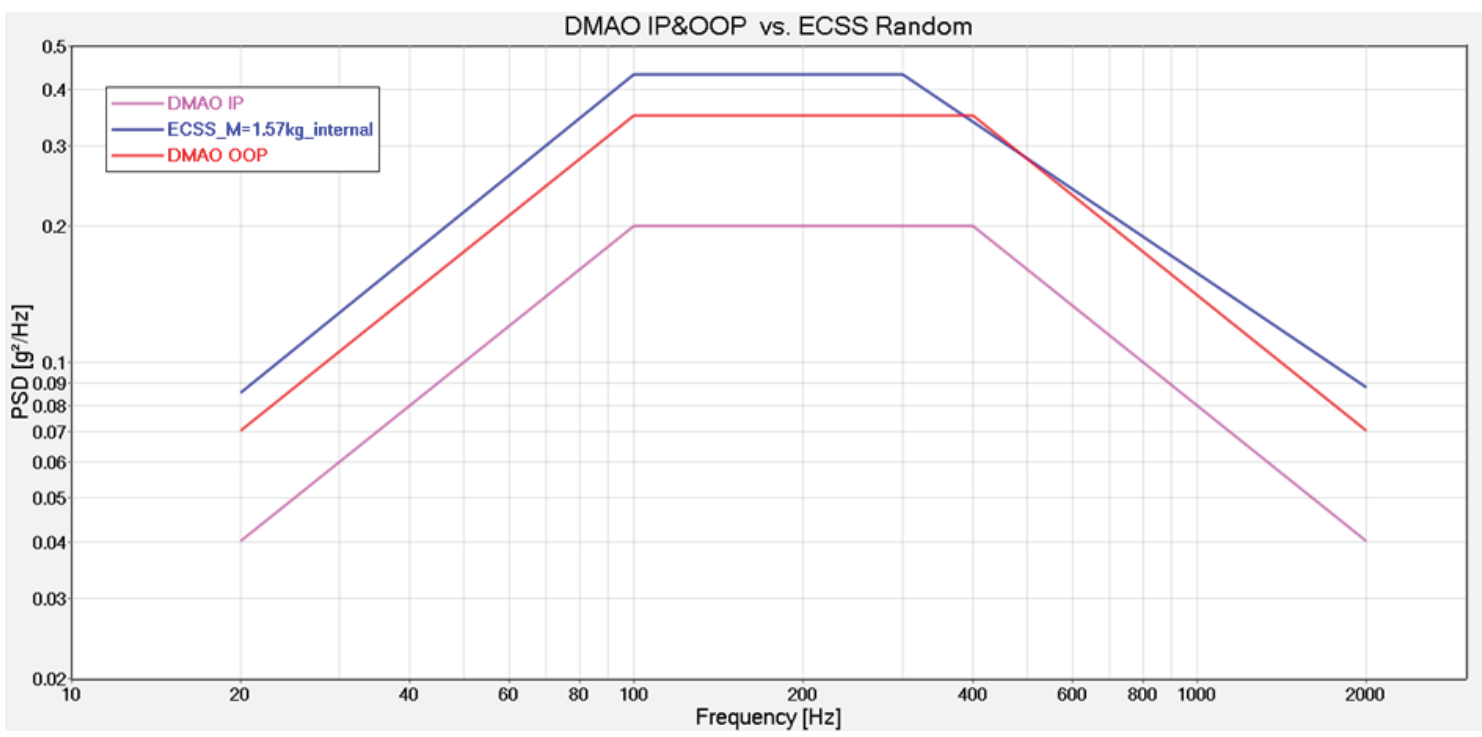

Figure 3. Loads for a $1.57 \mathrm{~kg}$ derived from ECSS 10, in-plane and out-of-plane (DMAO IP and DMAO OOP). 
These loads have compared to real loads of comparable mirrors from MTG and EnMap programmes. One fact that became obvious is that the generically derived load specification has a more flat shape in the low and high frequency domains as the real loads of MTG and EnMap. This has the consequence that more energy is introduced into the system whereas it is not of actual interest and does not bring more benefit. Therefore, we decided to steepen the ramp-up of the loads in the low frequency region and the ramp-down in the high frequency domain. The finally defined ramp-up is set with $4 \mathrm{~dB} / \mathrm{oct}$ and down with $5 \mathrm{~dB} /$ oct. An illustration is given in the next curves.

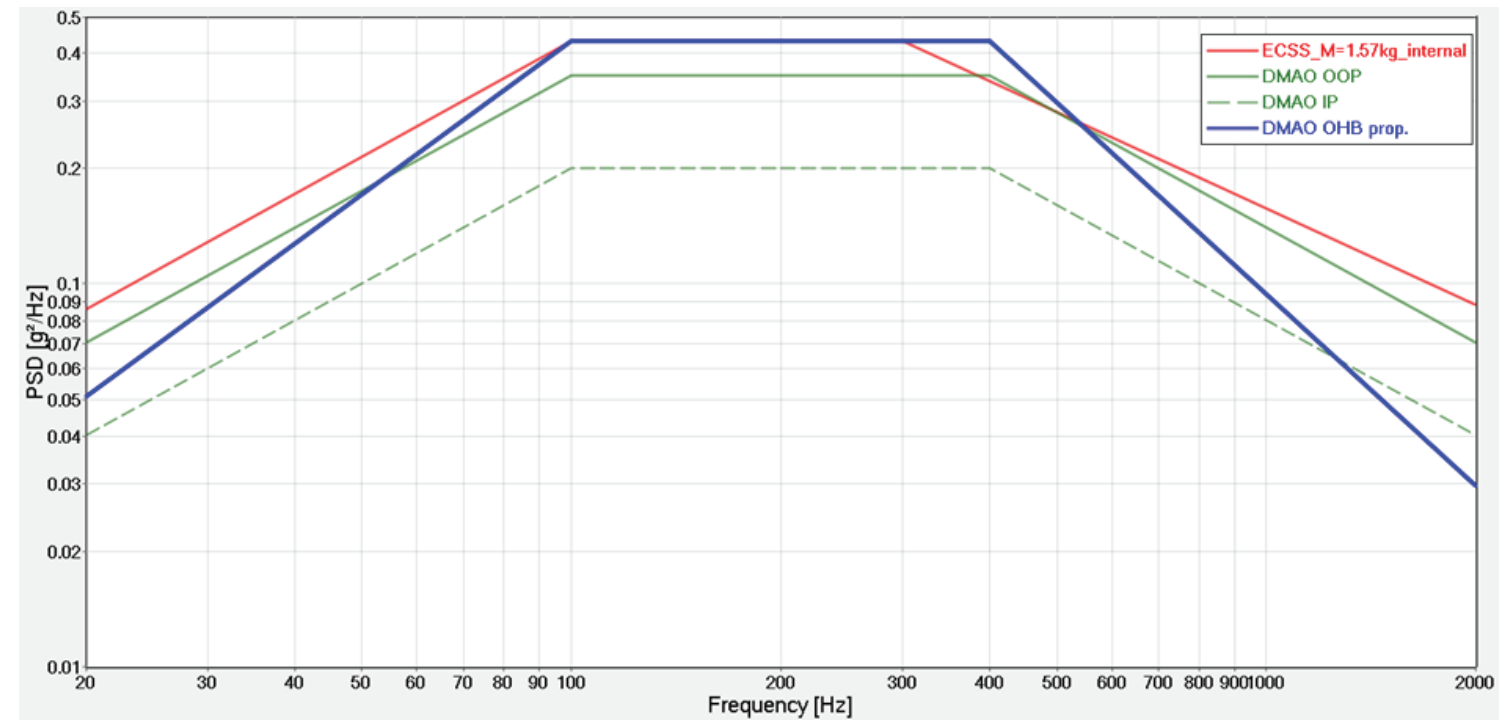

Figure 4. Loads for a $1.57 \mathrm{~kg}$ derived from ECSS [4]; Loads from former project for in-plane and out-of-plane (DMAO IP and DMAO OOP), Defined load level for requirement of this qualification campaign (DMAO OHB prop.).

In general, the generically derived loads from ECSS and the real curves of OHB experiences (MTG and EnMap) are mostly in line. Only the minor modification of a steeper ramp up and down was necessary. This resulted to the requirement on random loads given in the next table for the DM to be sustained without any damage or performance degradation.

Table 2. Specified random vibration level for DM testing.

\begin{tabular}{lr} 
Frequency $[\mathbf{H z}]$ & PSD $\left[\mathbf{g}^{2} / \mathbf{H z}\right]$ \\
\hline 20 & 0.05 \\
100 & 0.43 \\
400 & 0.43 \\
2000 & 0.03 \\
G $_{\text {rms }}$ & $17.92 \mathrm{~g}$
\end{tabular}

Shock loads play an important role for optical instrument since damage or rather failure of an optical component could result in the loss of the entire mission. Considering the critical aspects of shock loads and considering the risk of a total failure of the DM, it was considered necessary to verify the capability of the DM design to sustain shock loads by test.

To specify shock levels for the DM a few assumptions have been taken into account. As shock source a clamp band excitation (far-field environment) has been considered. Single point sources, like frangibolts or pyrotechnically actuated mechanisms were not taken into account since these sources and their locations are very specific to one particular platform and payload concept. The load path from the source (clamp band adapter) has been assumed to be larger than $1 \mathrm{~m}$. Reference shock response spectrum of EnMap and MTG IRS have both been used as basis [8][7]. The loads are applicable inside the optical instrument (MTG or EnMap). A damping factor of $\mathrm{Q}=10$ was applied for these applications. 
By considering these assumptions as well as real experience from these projects, finally a shock requirement was derived. This derived levels can be found in Figure 5 and has a maximum shock load at $300 \mathrm{~g}$ above $1000 \mathrm{~Hz}$.

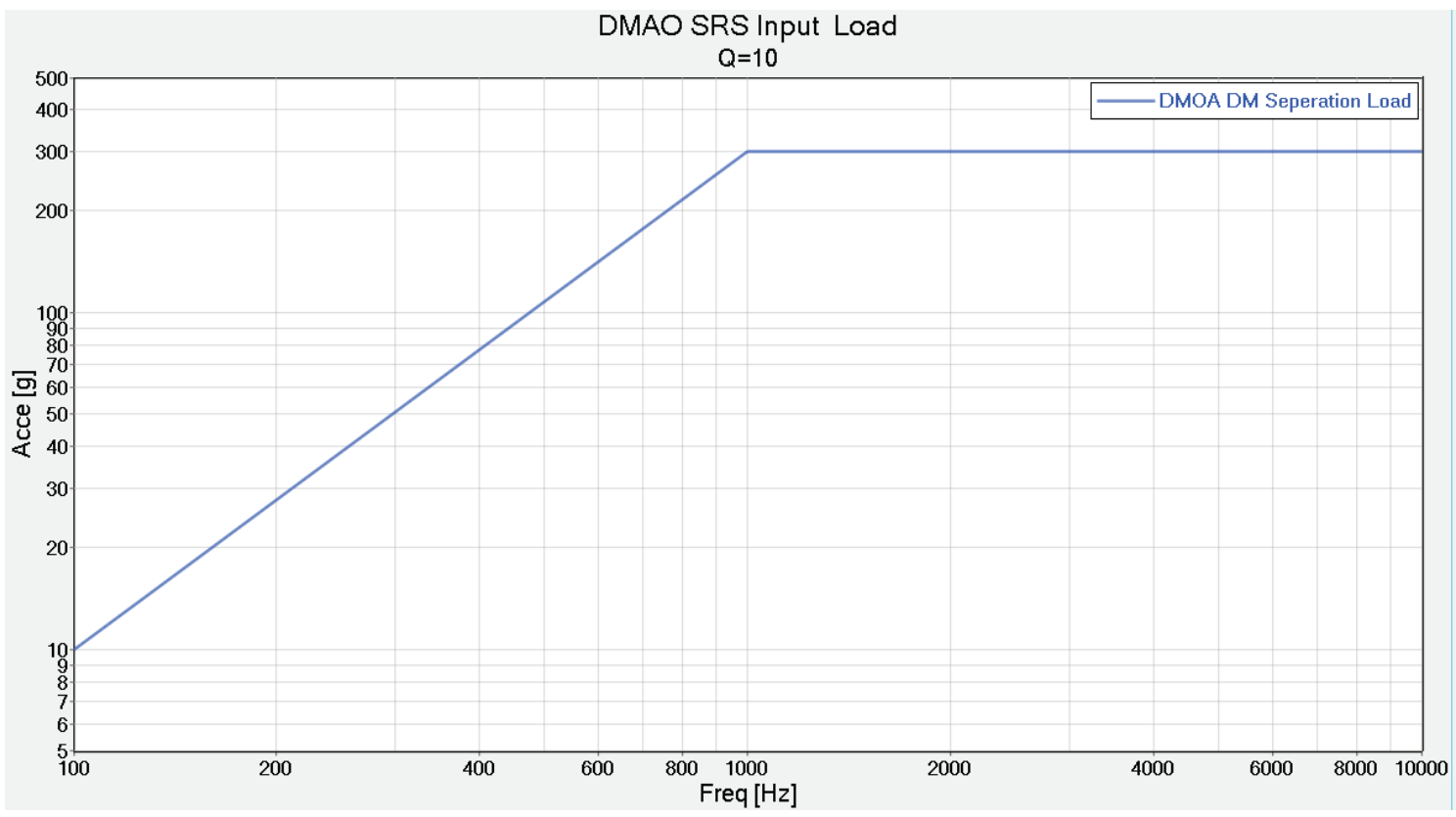

Figure 5. Specified and Tested Shock Levels.

Vibration tests (sinusoidal vibration and random vibration) have been successfully conducted in in-plane and out-of-plane directions with respect to the mirror surface. Sinusoidal vibration was performed with up to $20 \mathrm{~g}$ and random vibration with up to 17.8 gRMS.

\subsection{Thermal Requirements}

Considering the wide spectrum of potential applications of a DM also a wide range of possible operational temperatures is possible. For example if a DM is used in a science mission like EUCLID which is located at a Lagrange-Point, it would need to be operated at a very cold temperatures between roughly $120 \mathrm{~K}$ and $140 \mathrm{~K}$. If the DM would be used for a typical earth observation mission like MTG in a Geo-Stationary Orbit, operational temperatures are strongly influenced by the sun. Such operational temperature ranges of the optical components are typical between $-15^{\circ} \mathrm{C}$ and $+60^{\circ} \mathrm{C}$. The temperature limits are dominated by radiative exchange or the incoming sun light on the mirror (sun intrusion) [7]. Important to mention is that from $\mathrm{OHB}$ experience, it is not likely that a very wide temperature range (100K to $300 \mathrm{~K}$ ) for operational temperatures will occur within one mission only. The temperature set point will either be around a low temperature as for the example in EUCLID or to a higher temperature as for MTG [9][7]. However the alignment of instruments will be performed at room temperature what is to be considered for the definition of the temperature range and also the nonoperational temperatures need to be considered for survival. It might be considered to split the requirement in two applications low and higher temperature. However the thermal qualification of the DM of the previous GSTP showed that there was no problem from the large temperature range. The resulting thermal requirement was defined at that time such, that the DM shall fulfil the functional and performance requirements for the huge temperature range from $100 \mathrm{~K}$ to $333 \mathrm{~K}$ [3].

For various projects sun intrusion is a valid case and very stringent to the optical components as well. For example for the ADM Aeolus ALADIN instrument, a SiC mirror of $25 \mathrm{~mm}$ diameter was heated up to a temperature of $80^{\circ} \mathrm{C}$ in nonoperational mode [6]. For the MTG mission sun intrusion plays also an important role. Here a mirror with about the same size of the DM is exposed to sun illumination also within an operational mode. As a result, the mirror is heated up to $+60^{\circ} \mathrm{C}$. Of course the actual temperature of a mirror under sun illumination depends on the mirror size, reflectivity for the sun spectrum and most relevant on the thermal capacity and thermal conductivity of the material of substrate and mount. 
For the MTG FCI Instrument, the sun intrusion is about $100 \mathrm{~W} / \mathrm{cm}^{\wedge} 2$. However, the optical component is next to an intermediate focus in MTG, whereas for most of the applications of the DM, it will be place at a conjugated plane. This means that the intensity for the DM would be about a factor of 7 higher as for the primary mirror of the same telescope. Including margin, a factor of ten should be considered. The Solar constant is roughly $1350 \mathrm{~W} / \mathrm{m}^{\wedge} 2$ what corresponds to $0.135 \mathrm{~W} / \mathrm{cm}^{\wedge} 2$. Taking into account the factor derived from the examples, the solar intrusion which should be sustained by the $\mathrm{DM}$ is $1.35 \mathrm{~W} / \mathrm{cm}^{\wedge} 2$. A verification of the DM was done by analysis using COMSOL tool. It confirmed that the DM will sustain a sun intrusion of up to $1.5 \mathrm{~W} / \mathrm{cm}^{\wedge} 2 \mathrm{c}$.

\subsection{Dynamic Bandwidth}

The requirements on the dynamic bandwidth for DM actuation is quite difficult to define, since the needed frequency range strongly depends on the dedicated application. Hence, we split the regimes the DM can be used in the three categories: Static Phenomena, Low Frequency Phenomena and High Frequency Phenomena.

Static Phenomena are occurring only once in the lifetime of the mission. Compensation with a DM could be used for the following effects: manufacturing error correction, alignment, gravity release, compensation of CME-effects, alignment deterioration caused by slippage due to launch loads etc. Since these events occur only once, there is no specific requirement on the bandwidth.

Low-frequency Phenomena are disturbances with frequent occurrences like thermal drifts. This effects can easily be compensated with a DM. In order to compensate these kind of effects the correction chain shall have a slightly higher bandwidth as the effect itself. For usual thermal events the temporal constant is greater than 10 seconds, what would correspond to a frequency of $0.1 \mathrm{~Hz}$. Considering an application where the DM might be used as a scanning or refocus mechanism the frequency could be considered up to $10 \mathrm{~Hz}$.

High-frequency phenomena are potential application of a DM with an associated correction chain could also be used to compensate for micro-vibrations occurring in the instrument and other disturbing the optical Point Spread Function (PSF). Micro-vibrations are defined for a bandwidth of up to $500 \mathrm{~Hz}$. Compensating these vibrations would require an even higher bandwidth of the DM and correction chain. However, the compensation of high frequency phenomena like microvibrations is considered to be in the domain of "Adaptive Optics" which is beyond of the scope of this Active Optics activity. Furthermore, the optical aberrations caused by the micro-vibrations would be needed to be better understood before defining requirements for their compensation by a DM.

The maximum achieved closed loop actuation frequency of $19 \mathrm{~Hz}$ is compliant with the required frequency of $10 \mathrm{~Hz}$. The mirror was operated with an open loop frequency of $100 \mathrm{~Hz}$ which is compliant to the required specification for that activity. Nevertheless it is important to mention that for the conducted project the limiting factor of the achieved frequency is the driver electronics. This is a minor point, so that higher frequencies are easily achieved with the same technology.

\subsection{Radiation}

The radiation levels seen during a mission depend strongly on the instrument architecture and the mission and the orbit itself. Therefore, it is not straight forward to define a generic requirement for radiation for the DM. In the previous study the DM was tested by exposure to $\gamma$-irradiation and $\mathrm{p}+$-irradiation, yielded no visible degradation of the deformable mirror. The unpowered surface deformation as well as the stroke of the influence functions remained the same before and after irradiation. Therefore, it can be considered that the technology is not sensitive to radiation.

\section{VERIFICATION}

With the completion of the technology development activity presented here, we are proud to finally have at hand a fully qualified DM as a product. It demonstrated its suitability for a very wide field of applications and missions what is underlined by the successful testing. In the recent activities the focus was on a design adaption and the delta qualification for the structural requirements, consisting of sine, random and shock tests, whereas in the predecessor activity thermal and radiation testing was performed. More details on the design update and the successful results of the structural testing are described in another conference contribution on the subject provided by the Münster University of Applied Science in S. Leitz, et al. "Vibration and Shock Testing of a $50 \mathrm{~mm}$ Aperture Unimorph Deformable Mirror [2]. For a more detailed overview on the conducted testing and on the complete verification status, a dedicated verification matrix is given in the Appendix. A summary chart of achieved optical performance is presented below. 


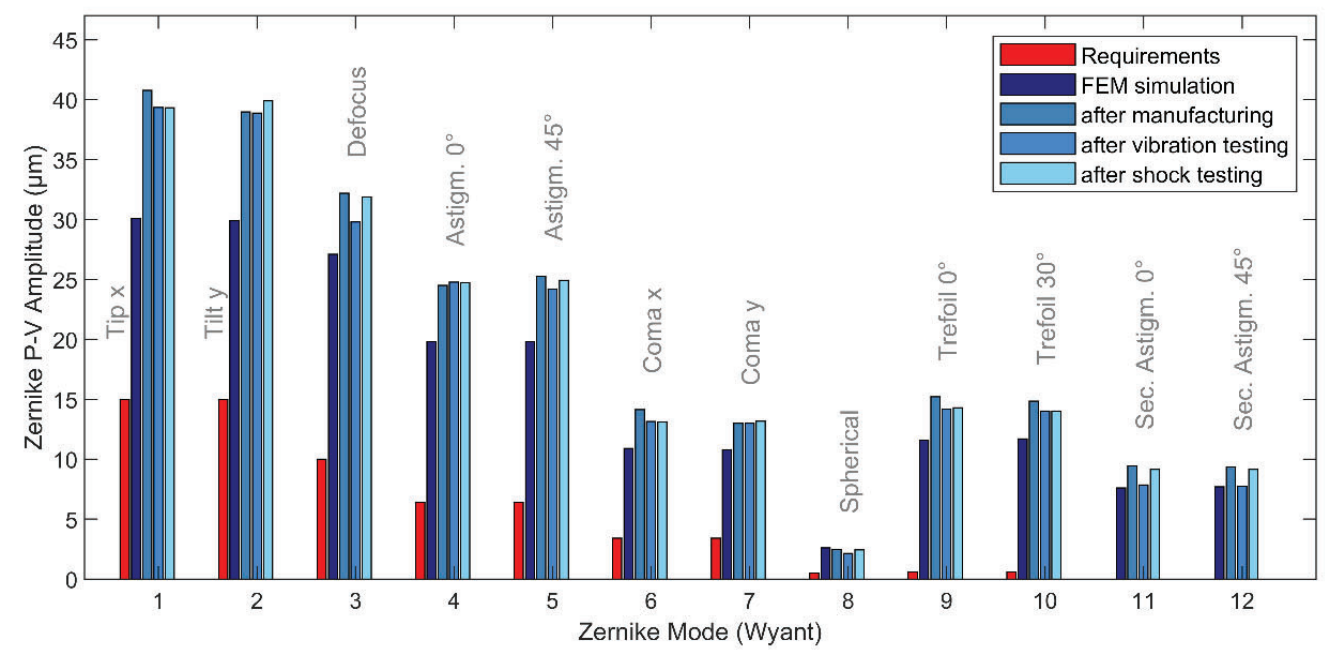

Figure 6. Zernike amplitudes of the Deformable Mirror measured after manufacturing, after the vibration test as well as after the shock test. The achieved performances are compared to the required performances and the predictions from the finite element simulation.

As visible in the plot all optical requirements regarding the strokes up to the Zernike mode 12 were overachieved by at least a factor of two. Furthermore, the strokes at the different verification steps were measured without change taken the measurements accuracies into account. Also these results demonstrate the high maturity level of the developed DM showing a strongly convincing and high potential technology.

\section{CONCLUSION}

Within the here presented GSTP project, a huge leverage of technology readiness was achieved. A major role played a consolidation and derivation of the requirements in the early stage of the development from a large variety but real space applications. It did provide a still challenging, but solid and realistic basis for the development and the subsequent definition of a suitable and reliable verification campaign, leading to a full qualification with its successful conduction. Hence, the viability of the technology was confirmed.

For the final TRL assessment we considered all conducted measures, the verification activities in relevant environment and the fact that all technological components were integrated. Thereby all criticalities which were identified in the previous study have been overcome successfully in this project. A technology readiness level (TRL) 5 is reached, as the component has been validated in relevant environment. Based on the high level of maturity, this deformable mirror is ready for the incorporation in all kinds of space optical instrument concepts. The achieved TRL of 5 is sufficient for the status of a PDR at payload level and gives thus a very good basis for all kinds of potential B2, C/D payload developments.

With the goal in mind to launch the next generation of optical space instruments with very large apertures and mirrors, the presented technology of a Deformable Mirror within an Active Optics correction chance will play a major role. This technology has the advantage that the current baseline design of the Deformable Mirror (DM) is well suited for a very large variety of possible instruments subjected to various environments and loads. To progress on the road of such large instruments with a primary mirror of up to $10 \mathrm{~m}$, some sequential steps are necessary for a full Active Optics Correction Chain. The roadmap and the associated steps are already defined on OHB side. They reach from enhancing the current design of the DM even further, to the preparation for an In-Orbit Demonstration (IOD) pushing the Technology Readiness Level finally up to 8 or 9 . 


\section{ACKNOWLEDGEMENT}

The University of Applied Sciences Münster and OHB System AG would like to acknowledge the constructive collaboration amongst all parties throughout the project and that this work was supported by ESA.

\section{APPENDIX}

Table 2. Specified random vibration level for DM testing.

\begin{tabular}{cc}
\hline Requirement Text & $\begin{array}{c}\text { Verification status } \\
\text { (Test, Analysis, } \\
\text { Review, Inspection) }\end{array}$ \\
\hline
\end{tabular}

Optical requirements

The DM shall have a usable aperture with a diameter of equal or larger than $50 \mathrm{~mm}$

Verified (T)

The DM shall enable a Tip/Tilt amplitude of equal to or higher than $0.3 \mathrm{mrad}$ for any operational condition.

The DM shall enable a Defocus (Z3) amplitude of higher than $5 \mu \mathrm{m}$ (defocus amplitude) for any operational condition.

Verified (T,A)

The DM shall enable the following Zernike amplitudes: Z1/Z2 7.5 $\mu \mathrm{m}, \mathrm{Z} 3: 5 \mu \mathrm{m}, \mathrm{Z} 4 / \mathrm{Z} 5: 3.2 \mu \mathrm{m}, \mathrm{Z6} / \mathrm{Z7}$ :

$1.7 \mu \mathrm{m}, \mathrm{Z} 8: 0.35 \mu \mathrm{m}, \mathrm{Z} 9 / \mathrm{Z} 10: 0.3 \mu \mathrm{m}$.

Verified (T,A)

The residual peak to valley wavefront for Zernike Z4 to Z10 in closed loop shall be smaller than $30 \mathrm{~nm}$.

Verified $(\mathrm{T}, \mathrm{A})$

The un-powered peak to valley deformation of the DM shall be smaller than $2 \mu \mathrm{m}$.

Verified (T)

The optical reflectivity of the DM at EOL shall be better than $95 \%$ for a wavelength from $500 \mathrm{~nm}$ to $13 \mu \mathrm{m}$.

If a laser application is applicable, the mirror shall guarantee full functionality and performance for a maximum optical input power of $5 \mathrm{~J} / \mathrm{cm}^{\wedge} 2(10 \mathrm{~ns}$ puls duration, $100 \mathrm{~Hz}$ repetition frequency, at wavelength $1064 \mathrm{~nm}$ ) for any operational condition.

\section{Structural Requirements}

The DM shall be designed to sustain without any degradation quasi-static loads up to $61 \mathrm{~g}$.

Verified (T,A,I)

The DM shall be designed to sustain without any degradation the sine loads up to $20 \mathrm{~g}$.

Verified (T,A,I)

The DM shall be designed to sustain without any degradation the random loads up to 17.82 gRMS.

Verified (T,A,I)

The DM shall be designed to sustain without any degradation the shock loads up to $300 \mathrm{~g}$ SRS.

Verified $(\mathrm{T}, \mathrm{A}, \mathrm{I})$

\section{Thermal Requirement}

The DM shall fulfil the functional and performance requirements as defined in this specification for a temperature range from $100 \mathrm{~K}$ to $333 \mathrm{~K}$.

Verified (T,A)

The DM shall sustain a sun intrusion of up to $1.5 \mathrm{~W} / \mathrm{cm}^{\wedge} 2$ considering the sun spectrum.

Verified (A)

The DM shall be designed to withstand the non-operational temperature range from $110 \mathrm{~K}$ to $353 \mathrm{~K}$ without any performance degradation.
Verified (T,A)

\section{Operational Requirements}




\begin{tabular}{|c|c|}
\hline $\begin{array}{l}\text { The DM shall fulfil the functional and performance requirements as defined in this specification for a } \\
\text { lifetime of } 8.5 \text { years operated with the specified closed loop frequency. }\end{array}$ & (A) \\
\hline $\begin{array}{l}\text { The DM shall be designed to withstand and fulfil performances for any atmospheric pressure between } \\
970 \text { mbar and } 1.33 \mathrm{E}-8 \mathrm{~Pa} \text {. }\end{array}$ & Verified $(\mathrm{T})$ \\
\hline $\begin{array}{l}\text { The DM shall be designed to have full functionality and performance for relative humidity between } \\
45 \% \text { and } 65 \% \text {. }\end{array}$ & Verified $(\mathrm{R})$ \\
\hline $\begin{array}{l}\text { The DM shall realize an open-loop bandwidth of up to 100Hz (@maximum stroke) fulfilling the } \\
\text { performance requirements as in this specification. }\end{array}$ & Verified $(\mathrm{T})$ \\
\hline $\begin{array}{l}\text { The DM shall realize a closed-loop bandwidth between } 0 \mathrm{~Hz} \text { and } 10 \mathrm{~Hz} \text { (@maximum stroke) fulfilling } \\
\text { the performance requirements as in this specification. }\end{array}$ & Verified $(\mathrm{T})$ \\
\hline The DM shall sustain the radiation levels without any degradation as specified in TBD. & Verified $(\mathrm{T})$ \\
\hline
\end{tabular}

\section{REFERENCES}

[1] Hallibert, P., "Enabling technologies for future large optical missions: current perspectives for astronomy and Earth observation at ESA," Proc. SPIE 10706, 221-235 (2018).

[2] S. Leitz, et al., "Vibration and shock testing of a $50 \mathrm{~mm}$ aperture unimorph deformable mirror," International Conference on Space Optics (ICSO), (2020).

[3] P. Rausch, S. Verpoort, "Performance verification and environmental testing of a unimorph deformable mirror for space applications," International Conference on Space Optics (ICSO) 2014, Tenerife, Spain (2014).

[4] European Space Agency ESTEC: Space engineering - Testing, "ECSS-E-ST-10-03C." ECSS, 1 June 2012.

[5] European Space Agency ESTEC: Spacecraft Mechanical Loads Analysis Handbook, "ECSS-E-HB-32-26A." ECSS, 19 February 2013.

[6] European Space Agency, "Aeolus - Aladin.” https://earth.esa.int/eogateway/instruments/aladin (18 February 2021).

[7] Eumetsat, „Meteosat Third Generation.“ https://www.eumetsat.int/meteosat-third-generation (18 February 2021).

[8] Deutsche Luft und Raumfahrtgesellschaft, „EnMap - Die erste deutsche Hyperspektral-Satellitenmission.“ https://www.dlr.de/rd/desktopdefault.aspx/tabid-2440/3586_read-28911/ (18 February 2021).

[9] European Space Agency, "Euclid Mission - Telescope.” Slate 19 September 2019, https://sci.esa.int/web/euclid//telescope (18 February 2021). 